\title{
Using Health Information Technology to Prevent and Treat Diabetes
}

\author{
Neal Kaufman ${ }^{1}$ and Rachel R. Bian ${ }^{2}$
}

\section{Introduction}

$\mathbf{F}$

OR THIS YEAR'S ARTICLE ON INFORMATION TECHNOLOGY, we selected articles that will give the reader a sense of the current state of the art of the field and a hint of where it is going. To help organize thinking about how technology can improve outcomes for people with or at risk for diabetes, it is helpful to frame technology's roles in the range of approaches that are being used to improve outcomes.

A new equilibrium is being forged among independent, but overlapping, health-promoting paradigms for which information technology often is at the core of any approach. There are four tried and true approaches that complement each other but also compete for resources as well as attention.

Personal health approaches: Those services and supports that are provided by an organized healthcare system primarily for the benefit of the individual receiving the service.

Public health approaches: Activities aiming to provide conditions in which people can be healthy that focus on entire populations rather than on individual patients or diseases. Public health is concerned with the total system and not only the eradication of a particular disease.

Direct-to-consumer approaches: Goods and services marketed and sold directly to end users and that are usually paid for by the consumer.

Population health approaches: Improving health outcomes of one or more subpopulations out of the entire population is the area of focus. It is understood that such population health outcomes are the product of multiple determinants of health. The outcomes are influenced by social, economic, and physical environments; personal health and lifestyle practices; individual capacity and coping skills; human biology; prenatal and early childhood development; and healthcare services.

These subpopulations can be geographic regions, such as nations or communities, but they can also be other groups, such as employees, ethnic groups, disabled persons, members of a health plan, patients being served by a healthcare system, or individuals with specific clinical conditions or life cir- cumstances. Often groups are segmented by risk or health status (e.g., stable and well, moderate risk, high risk/high cost). Most of the articles chosen for this chapter would fall into population health approaches that are linked to, or integrated with, personal health approaches.

As we think about ways to improve outcomes, there are six key questions that must be answered in order for the chosen approach to have a chance of being successful.

1. How is the target population defined and segmented from the larger population?

2. What are the characteristics of the target population?

3. What are the key modifiable determinants of the health status and healthcare utilization patterns of the target population?

4. What are the evidence-based approaches that have been shown to modify the identified determinants in the target population?

5. Can these approaches be cost-effectively and efficiently provided at a large enough scale to improve the entire population's outcomes?

6. Is there capacity to successfully deploy the chosen approaches?

Fundamentally, to improve outcomes for people with, or at risk for, diabetes, we must focus on two independent but interrelated areas:

1. Helping individuals improve their lifestyle behaviors (e.g., eating better, being more active, sleeping enough, not smoking, practicing safe sex, maintaining friendships)

2. Helping individuals better self-manage their chronic conditions

There are many programs addressing these areas with effectiveness backed by decades of accumulated evidence. The challenge is getting them to the right person, at the right time, with the right degree of intensity. It is hoped that this review will supply the reader with information about a number of successful and scalable approaches.

\footnotetext{
${ }^{1}$ UCLA Schools of Medicine and Public Health, Los Angeles, CA

${ }^{2}$ University of Michigan Medical School, Ann Arbor, MI.
} 


\section{Effects of a web-based tailored multiple-lifestyle intervention for adults: a two-year randomized controlled trial comparing sequential and simultaneous delivery modes}

Schulz $D N^{1}$, Kremers SPJ ${ }^{2}$, Vandelanotte $C^{3}$, van Adrichem $M J G^{1}$, Schneider $F^{1}$, Candel $M J J M^{4}$, de Vries $H^{1}$

${ }^{1}$ Department of Health Promotion, CAPHRI School for Public Health and Primary Care, Maastricht University, Maastricht, The Netherlands; ${ }^{2}$ Department of Health Promotion, Nutrition and Toxicology Research Institute Maastricht (NUTRIM), Maastricht University, Maastricht, The Netherlands; ${ }^{3}$ Institute for Health and Social Science Research, Centre for Physical Activity Studies, Central Queensland University, Rockhampton, Australia; and ${ }^{4}$ Department of Methodology and Statistics, CAPHRI School for Public Health and Primary Care, Maastricht University, Maastricht, Netherlands

J Med Internet Res 2014; 16: e26

Note from author: Since these two articles are from the same study, the abstract and comments have been combined into one piece which can be found below.

\section{Economic evaluation of a web-based tailored lifestyle intervention for adults: findings regarding cost-effectiveness and cost-utility from a randomized controlled trial}

Schulz DN ${ }^{1}$, Smit ES ${ }^{1,2}$, Stanczyk $N E^{1}$, Kremers $S P J^{3}$, de Vries $H^{1}$, Evers SMAA ${ }^{4}$

${ }^{1}$ Department of Health Promotion, CAPHRI School for Public Health and Primary Care, Maastricht University, Maastricht, The Netherlands; ${ }^{2}$ Department of Communication Science, Amsterdam School of Communication Research/ASCoR, University of Amsterdam, Amsterdam, The Netherlands; ${ }^{3}$ Department of Health Promotion, Nutrition and Toxicology Research Institute Maastricht (NUTRIM), Maastricht University, Maastricht, The Netherlands; and ${ }^{4}$ Department of Health Services Research, CAPHRI School for Public Health and Primary Care, Maastricht University, Maastricht, The Netherlands

J Med Internet Res 2014; 16: e91

\section{Background}

Different studies have reported the effectiveness of web-based computer-tailored lifestyle interventions, but combined impact and economic evaluations of these interventions are rare. Doing such evaluations are critical to the ability of effective programs to go to scale. Computertailored interventions are an interesting and promising option to make the healthcare system more sustainable. The benefits of such approaches derive from proven clinical effectiveness. Also important is their potential cost-effectiveness and cost-savings due to relatively low intervention costs and wide reach. Information regarding the impact and cost-effectiveness of web-based computertailored intervention programs is crucial for healthcare decision makers and governments seeking to make evidence-based decisions regarding large-scale implementation of such programs.

\section{Methods}

The aim of the study was to assess the impact on individuals, and from a societal perspective the cost-effectiveness and costutility of two different versions of a web-based computertailored lifestyle intervention for adults compared to a control group that received only a minimal intervention. The economic evaluation was part of a 2-year randomized controlled trial including 3 study groups (control, sequential conditions, and simultaneous conditions). All groups received personalized health risk appraisals based on the guidelines for physical activity, fruit intake, vegetable intake, alcohol consumption, and smoking. Additionally, participants in the sequential condition group $(N=1736)$ received personal advice about one lifestyle behavior in the first year and a second behavior in the second year; participants in the simultaneous condition group $(N=1638)$ received personal advice about all unhealthy behaviors in both years. During a period of 24 months, healthcare utilization, medication use, absenteeism from work, and quality of life (EQ-5D-3L) were assessed every 3 months using web-based questionnaires. Demographics were assessed at baseline, and lifestyle behaviors were assessed at both baseline and after 24 months. Cost-effectiveness and cost-utility analyses were performed based on the outcome measures lifestyle factor (the number of guidelines respondents adhered to) and quality of life, respectively. Incremental costs (in Euros) and effects were calculated for all 3 study groups. A net monetary benefit was also calculated by valuing the effectiveness and utility outcomes in monetary values using a threshold for society's willingness to pay (WTP) per gain in lifestyle factors (i.e., per additional guideline met) and per QALY gained.

\section{Results}

Both tailoring strategies were associated with small selfreported behavioral changes. The sequential condition had the most significant effects compared to the control condition after 12 months (effect size $=0.28$ ). After 24 months, the simultaneous condition was most effective (effect size $=$ 0.18). All 5 individual lifestyle behaviors changed over time, but few effects differed significantly between the conditions. At both follow-ups, the sequential condition had significant changes in smoking abstinence compared to the simultaneous condition (12-month effect size $=0.31 ; 24$ month effect size $=0.41$ ). The sequential condition was more effective in decreasing alcohol consumption than the control condition at 24 months (effect size $=0.27$ ). Change was predicted by the amount of exposure to the intervention (total visiting time: beta $=-.06 ; p=.01$; total number of visits: beta $=-.11 ; p<.001)$. Both interventions were appreciated well by respondents without significant differences between conditions.

A total of 1733 participants were included in the economic analyses. From a society's willingness to pay of $€ 4594$ per additional guideline met, the sequential intervention $(n=552)$ was likely to be the most cost-effective, whereas from a society's willingness to pay of $€ 10,850$, the simultaneous intervention $(n=517)$ was likely to be most cost-effective. The control condition $(n=664)$ appeared to be preferred with regard to quality of life. 


\section{Conclusions}

Although both programs proved to be effective, no definite finding could be drawn as their effectiveness. The authors suggest that the best mode of intervention may depend on the behavior that is targeted or on personal preferences and motivation. Further research is needed to identify moderators of intervention effectiveness. The results need to be interpreted in view of the high and selective dropout rates, multiple comparisons, and modest effect sizes. However, a large number of people were reached at low cost and behavioral change was achieved after 2 years.

Both the sequential and the simultaneous lifestyle interventions were quite cost-effective when it related to the lifestyle factor, whereas the control condition was costeffective when it concerned quality of life. However, since there is no accepted cutoff point for the willingness to pay per gain in lifestyle behaviors, it is impossible to draw strong conclusions. There is a need for more economic evaluations of lifestyle intervention.

\section{Comment}

It is encouraging to see that web-based programs can demonstrate positive behavior change and economic benefits. While it was somewhat discouraging to not see a greater overall effect, this study once again demonstrates how hard it is to show results that matter. Perhaps the wrong question was asked. Maybe the difference between sequential and simultaneous delivery isn't that important. Perhaps the quality and intensity of the intervention is more important. I would guess that approaches that provide more long-term engagement would be more successful.

Attempting to prove the cost-effectiveness and costbenefit of web-based interventions is critical to the field. Without these types of analysis, effective programs will not be able to go to scale. While the ability to improve health and save money is the hallmark of a commercially successful approach, there are many other outcomes that need to be assessed and valued depending on the exact circumstances of the program's deployment. Other key areas include improving the quality of care provided; enhancing patient/health plan member satisfaction; driving uptake of other important services and supports; improving the communication and relationships with providers in a provider network; and responding to the expectation of the entity's customer (i.e., payer or employer). It is critical that these and other outcomes are assessed and reached if there are to be widespread adoption of educational services and supports by healthcare providers, payers, or employers.

\section{Telemedicine application in the care of diabetes patients: systematic review and meta-analysis}

Marcolino $\mathrm{MS}^{1,2}$, Maia $\mathrm{JX}^{2}$, Alkmim $M B M^{2}$, Boersma $E^{3}$, Ribeiro $A^{1,2}$

${ }^{I}$ Medical School, Universidade Federal de Minas Gerais, Belo Horizonte, Brazil; ${ }^{2}$ University Hospital, Universidade Federal de Minas Gerais, Belo Horizonte, Brazil; and ${ }^{3}$ Erasmus MC, Rotterdam, The Netherlands

\section{Background}

Telemedicine programs for patients with diabetes have increased in number, but insufficient research has been done to demonstrate if these approaches improve care, improve health outcomes, and/or decrease cost. The objective of this study is to conduct a systematic review and meta-analysis to document the impact of telemedicine on patients with diabetes.

\section{Methods}

A comprehensive literature search identified randomized controlled trials (RCTs) assessing the impact of telemedicine interventions on change in hemoglobin A1c (HbA1c), blood pressure, LDL cholesterol (LDL-c), and body mass index (BMI) in diabetes patients. The strategies of telemedicine application included in this review were computerized systems for information exchange, video conferencing, and exchange of information via telephone or other mobile devices, short message service, or through the Internet. Reported outcomes include HbA1c, blood pressure, BMI, and LDL-c. Studies utilized personalized feedback from healthcare providers to the patient or other healthcare provider.

\section{Results}

Fifteen articles with 13 studies were included, evaluating a total of 4207 outpatient participants. Telemedicine was associated with $-0.44 \%$ reduction $(95 \% \mathrm{CI}-0.61$ to $-0.26 \%$; $p<0.001)$ in $\mathrm{HbA} 1 \mathrm{c}(-4.8 \mathrm{mmol} / \mathrm{mol})$ compared to usual care, and this effect is more pronounced in type 1 diabetes patients. Among studies evaluating effect of telemedicine on LDL-c, there was a reduction of $6.6 \mathrm{mg} / \mathrm{dL}(95 \% \mathrm{CI}-8.3$ to $-4.9 \mathrm{mg} / \mathrm{dL} ; p<0.001)$. Among those evaluating effect on blood pressure, there was no significant effect due to telemedicine. Only 2 studies evaluated effect on BMI and they did not show any significant decrease.

\section{Conclusions}

This meta-analysis revealed that use of telemedicine within diabetes care is associated with reductions in $\mathrm{HbA1c}$ levels, but is not associated with clinically significant reductions in blood pressure, LDL-c, or BMI. Further studies are needed to explore the possibility of using telemedicine to improve diabetes care outcomes.

\section{Comment}

This study attempts to summarize the literature on the impact of "Internet" or "telemedicine" interventions on patients with diabetes. It may still be too early to tell if telemedicine approaches are able to change outcomes and/or lower costs. We fully expect that they will, but the challenge will be to understand which patients, with which behaviors and which complications, will most likely benefit from which intervention. As more is known, we have no doubt that interventions tailored to the unique characteristics of each patient will have a significant effect. The bigger question is whether creators of these approaches will develop sustainable business models that allow successful programs to go to scale 
so that we can make a real impact at a population health level. While this study can help point us in the right direction, it suffers from the limitations of this type of review and the limitations of meta-analysis. We may never have indisputable proof of efficacy but that shouldn't surprise us or stop us. Just as beauty is in the eyes of the beholder, so too is effectiveness. Each of us sees different outcomes as being more relevant or, perhaps better said, more important. The key is to continue to innovate, evaluate, and modify based on the results if one really wants to see impact.

\section{Diabetes self-management smartphone application for adults with type 1 diabetes: randomized controlled trial}

Kirwan $M^{l}$, Vandelanotte $C^{l}$, Fenning $A^{l}$, Duncan $M J^{1}$

${ }^{1}$ Institute for Health and Social Science Research, Central Queensland University, North Rockhampton, Australia

J Med Internet Res 2013; 15: e235

\section{Background}

Persistently poor glycemic control in adult type 1 diabetes patients is a common, complex, and serious problem initiating significant damage to the cardiovascular, renal, neural, and visual systems. Currently, there are numerous low-cost and free diabetes self-management smartphone applications available in online stores. Their effectiveness is unproven. The aim of this study was to examine the effectiveness of a freely available smartphone application combined with textmessage feedback from a certified diabetes educator to improve glycemic control and other diabetes-related outcomes in adult patients with type 1 diabetes.

\section{Methods}

The authors performed a two-group randomized controlled trial. Patients were recruited through an online type 1 diabetes support group and letters mailed to adults with type 1 diabetes throughout Australia. In a 6-month intervention, followed by a 3-month follow-up, patients $(n=72)$ were randomized to usual care (control group) or usual care and the use of a smartphone application (Glucose Buddy) with weekly textmessage feedback from a Certified Diabetes Educator (intervention group). All outcome measures were collected at baseline and every 3 months over the study period. Patients' glycosylated hemoglobin levels (HbA1c) were measured with a blood test, and diabetes-related self-efficacy, self-care activities, and quality of life were measured with online questionnaires.

\section{Results}

The mean age of patients was 35.2 years (SD 10.43) (28 male, 44 female). Patients had been diagnosed with type 1 diabetes for a mean of 18.94 years (SD 9.66). Of the initial 72 patients, 53 completed the study (25 intervention, 28 control group). The intervention group significantly improved glycemic control (HbA1c) from baseline (mean 9.08\%, SD 1.18) to 9 -month follow-up (mean $7.80 \%$, SD 0.75 ), compared to the control group (baseline: mean $8.47 \%$, SD 0.86, follow-up: mean $8.58 \%$, SD 1.16). No significant change over time was found in either group in relation to self-efficacy, self-care activities, and quality of life.

\section{Conclusions}

In addition to the usual care, the use of a diabetes-related smartphone application together with weekly text-message support from professional health caregivers can significantly improve glycemic control in adults with type 1 diabetes.

\section{Comment}

This small study is encouraging regarding changes in HbA1c though discouraging in terms of improving some patient attitudes and behaviors. A larger study would be necessary to document the generalizability of any of the results. We are not surprised that the more difficult to improve self-efficacy, self-care activities, and qualityof-life changes weren't demonstrated. Making a lasting impact on these and other psychosocial outcomes would probably require a more intensive and robust intervention. I am sure that those approaches are being studied and expect/hope that they will demonstrate results soon.

\section{Mobile applications for diabetics: a systematic review and expert-based usability evaluation considering the special requirements of diabetes patients age $\mathbf{5 0}$ years or older}

Arnhold M, Quade M, Kirch W

Research Association Public Health Saxony and SaxonyAnhalt, Medizinische Fakultät Carl Gustav Carus, Technische Universität Dresden, Dresden, Germany

J Med Internet Res 2014; 16: e104

\section{Background}

Many mobile health (mhealth) applications (apps) have been developed in recent years to support effective selfmanagement of patients with diabetes mellitus type 1 or 2 . Their effectiveness and appropriateness for patient use has not adequately been evaluated. Given the extraordinary low barrier to entry (cost and complexity of developing app is quite low), it is even more important to get a solid understanding of the benefits and liabilities for patients who use apps.

\section{Methods}

The authors carried out a systematic review of all currently available diabetes apps for the operating systems iOS and Android. They considered the number of newly released diabetes apps, range of functions, target user groups, languages, acquisition costs, user ratings, available interfaces, and the connection between acquisition costs and user ratings. They also focused on whether the available applications serve the special needs of diabetes patients aged 50 or older by performing an expert-based usability evaluation based on a representative $10 \%$ sample of diabetes apps. 


\section{Results}

In total, the authors analyzed 656 apps and found that 355 $(54.1 \%)$ offered just one function and $348(53.0 \%)$ provided a documentation function. The dominating app language was English $(85.4 \%, 560 / 656)$, patients represented the main user group $(96.0 \%, 630 / 656)$, and the analysis of the costs revealed a trend toward free apps $(53.7 \%, 352 / 656)$. The median price of paid apps was $€ 1.90$. The average user rating was 3.6 stars (maximum 5). The analyses indicated no clear differences in the user rating between free and paid apps. Only $30(4.6 \%)$ of the 656 available diabetes apps offered an interface to a measurement device. The authors evaluated 66 apps within the usability evaluation. On average, apps were rated best regarding the criterion "comprehensibility" (4.0 out of 5.0), while showing a lack of "fault tolerance" (2.8 out of 5.0). Of the 66 apps, $48(72.7 \%)$ offered the ability to read the screen content aloud. The number of functions was significantly negative correlated with usability. The presence of documentation and analysis functions reduced the usability score significantly by 0.36 and 0.21 points respectively.

\section{Conclusions}

A great number of diabetes apps already exist, but the majority offer similar functionalities and combine only one to two functions in one app. Patients and physicians alike should be more involved in the app development process. The authors expect that the data transmission of health parameters to physicians will gain more importance in future applications. The usability of diabetes apps for patients aged 50 or older was moderate to good. But this result applied mainly to apps offering a small range of functions. Multifunctional apps were used in much smaller scale. Moreover, the presence of a documentation or analysis function resulted in significantly lower usability scores. The operability of accessibility features for diabetes apps was quite limited, except for the feature "screen reader."

\section{Comment}

One thing for sure is that there are a plethora of applications claiming to provide education and support to individuals with diabetes. The overwhelming challenge is that very few of them actually have been demonstrated to help. We must be patient. After all, these applications have mostly been built with a single function in mind (e.g., track steps or blood glucose, communicate with a peer, receive reminders, ask an expert a question). It will only be when these apps are integrated into wellconceived and integrated approaches can we expect to see demonstrable and significant changes in outcomes.

\section{Web 2.0 chronic disease self-management for older adults: a systematic review}

Stellefson $M^{1}$, Chaney $B^{l}$, Barry $A E^{l}$, Chavarria $E^{l}$, Tennant $B^{1}$,Walsh-Childers $K^{2}$, Sriram $P S^{3}$, Zagora $J^{4}$

${ }^{1}$ Department of Health Education and Behavior, Center for Digital Health and Wellness; ${ }^{2}$ Department of Journalism; ${ }^{3}$ Department of Medicine; and ${ }^{4}$ Department of Health Education and Behavior, University of Florida, Gainesville, FL

J Med Internet Res 2013; 15: e35

\section{Background}

Participatory Web 2.0 interventions promote collaboration to support chronic disease self-management. (Note that Web 1.0 interventions are those that primarily make written and audio materials available online; Web 2.0 interventions utilize a fuller range of Internet services such as contributing to or consuming user-generated health content including blog posts, hospital or doctor reviews, and podcasts; interacting with digital coaches; participating in online discussion boards or online self-help groups; using multimedia-sharing software to share disease management videos, wikis, and podcasts; and using teleconferencing tools such as Skype provide intimate, two-way communication channels). Growth in Web 2.0 interventions has led to the emergence of e-patient communication tools that enable older adults to locate and share disease management information and receive interactive healthcare advice. To date, there are no review articles investigating the planning, implementation, and evaluation of Web 2.0 chronic disease self-management interventions for older adults (mean age $\geq 50$ ) with one or more chronic disease(s).

\section{Methods}

A systematic literature search was conducted using six popular health science databases. The RE-AIM (Reach, Efficacy, Adoption, Implementation, and Maintenance) model was used to organize findings and compute a study quality score (SQS) for 15 reviewed articles.

\section{Results}

Most interventions were adopted for delivery by multidisciplinary healthcare teams and tested among small samples of white females with diabetes. Studies indicated that Web 2.0 participants felt greater self-efficacy for managing their disease(s) and benefited from communicating with healthcare providers and/or website moderators to receive feedback and social support. Participants noted asynchronous communication tools (e.g., e-mail, discussion boards) and progress tracking features (e.g., graphical displays of uploaded personal data) as being particularly useful for selfmanagement support. Despite high attrition being noted as problematic, this review suggests that greater Web 2.0 engagement may be associated with improvements in health behaviors (e.g., physical activity) and health status (e.g., HRQoL). However, few studies indicated statistically significant improvements in medication adherence, biological outcomes, or healthcare utilization. Mean SQS scores were notably low $($ mean $=63 \%$, SD $18 \%)$. Studies were judged to be weakest on the maintenance dimension of RE-AIM; 13 reviewed studies $(87 \%)$ did not describe any measures taken to sustain Web 2.0 effects past designated study time periods. Detailed process and impact evaluation frameworks were also missing in almost half $(n=7)$ of the reviewed interventions.

\section{Conclusions}

There is need for a greater understanding of the costs and benefits associated with using patient-centered Web 2.0 technologies for chronic disease self-management. More research is needed to determine whether the long-term effectiveness of these programs is sustainable among larger, more diverse samples of chronically ill patients. The effective 
translation of new knowledge, social technologies, and engagement techniques will likely result in novel approaches for empowering, engaging, and educating older adults with chronic disease.

\section{Comment}

This study quite effectively demonstrates some of the benefits from advanced Internet functionalities on patient outcomes and satisfaction. The effectiveness of any of these interventions, and the components they contain, is based on a variety of elements. These include the appropriateness of foundational theories on which the program is based for the specific target population; the trustworthiness and reputation of the "sponsor" of the website; the usability of the user interfaces; the degree to which individuals are engaged and touch the website over time; the specifics of the content presented and its ability to influence the participant; the support the participant can receive beyond the website; the contextual influences on a person's ability to adopt and sustain new behaviors over time; and more. Despite all the complexity of these efforts, they are quite promising and give us hope for a future in which more individuals will be able to get the education and support they need, when and where they need it.

\section{Computer-based interventions to improve self-management in adults with type 2 diabetes: a systematic review and meta-analysis}

Pal $K^{1}$, Eastwood $S V^{2}$, Michie $S^{3}$, Farmer $A^{4}$, Barnard $M L^{5}$, Peacock $R^{6}$, Wood $B^{7}$, Edwards $P^{8}$, Murray $E^{1}$

${ }^{1}$ UCL Research Department of Primary Care and Population Health, University College London, London, UK; ${ }^{2} I n-$ ternational Centre for Circulatory Health, Imperial College, London, UK; ${ }^{3}$ Department of Clinical, Educational and Health Psychology, University College, London, UK; ${ }^{4}$ Department of Primary Care Health Sciences, University of Oxford, Oxford, UK; ${ }^{5}$ Department of Diabetes, The Whittington Hospital NHS Trust, London, UK; ${ }^{6}$ Archway Healthcare Library, London, UK; ${ }^{7}$ Diabetes Self-Management Program (DSMP), Co-creating Health, London, UK; and ${ }^{8}$ Department of Population Health, London School of Hygiene \& Tropical Medicine, London, UK

Diabetes Care 2014; 37: 1759-66

\section{Background}

Structured patient education programs can reduce the risk of diabetes-related complications. However, people appear to have difficulties attending face-to-face education. Other delivery channels are needed but research supporting their use is limited.

\section{Methods}

This review looked at the impact of computer-based diabetes self-management interventions on health status, cardiovascular risk factors, and quality of life of adults with type 2 diabetes. The authors did a comprehensive literature search to find relevant articles.

\section{Results}

Sixteen randomized controlled trials with 3,578 participants met the inclusion criteria. Interventions were delivered via clinics, the Internet, and mobile phones. Computer-based diabetes self-management interventions appear to have small benefits on glycemic control: the pooled effect on HbA1c was $20.2 \%$ (22.3 $\mathrm{mmol} / \mathrm{mol}$ [95\% CI $20.4 \%$ to $20.1 \%$ ]). A subgroup analysis on mobile phone-based interventions showed a larger effect: the pooled effect on $\mathrm{HbA} 1 \mathrm{c}$ from three studies was $20.50 \%$ ( $25.46 \mathrm{mmol} / \mathrm{mol}$ [ $95 \%$ CI $20.7 \%$ to $20.3 \%$ ]). There was no evidence of improvement in depression, quality of life, blood pressure, serum lipids, or weight. There was no evidence of significant adverse effects.

\section{Conclusions}

Computer-based diabetes self-management interventions to manage type 2 diabetes appear to have a small beneficial effect on blood glucose control, and this effect was larger in the mobile phone subgroup. There was no evidence of benefit for other biological, cognitive, behavioral, or emotional outcomes.

\section{Comment}

This study, while presenting discouraging results, should not deter us from hoping that new studies will demonstrate impact. All reviewed interventions were published before November 2011 and were therefore probably implemented in 2009-2010 and most likely developed in 2007-2008; 2007 is a very, very long time ago in information technology development. Web 2.0 was just beginning and the sophistication of the technologyenabled interventions was low. There are several key elements to success that more modern interventions possess. These include enhanced patient engagement, ability to self-tailor the experience, linkages to uploadable sensing devices, social networking, etc. Fortunately, the pace of innovation is quite rapid. Unfortunately, the pace of research and publication about innovations is quite slow. This is all the more important because often reimbursement follows high-quality studies that demonstrate efficacy. Also, most reviews, including this one, omit studies that were not randomized and controlled. While RCTs are the gold standard, often by the time an RCT is completed and published, the intervention's technology may already be obsolete, or at least not state of the art. This is a challenge that is not easy to solve but "good enough" research methods (often using econometric approaches without random assignment) can provide highly valuable and actionable conclusions. After all, most economists do not have the benefit of randomized controlled studies. If anyone wants to give me and a number of other people $\$ 1$ million and see what happens...be my guest.

\section{Effects of diabetes self-management programs on time-to-hospitalization among patients with type 2 diabetes: a survival analysis model}

\footnotetext{
Adepoju $O E^{l}$, Bolin $J N^{1}$, Phillips $C D^{1}$, Zhao $H^{2}$, Ohsfeldt $R L^{1}$, McMaughan DK', Helduser $J W^{1}$, Forjuoh $\mathrm{SN}^{1,3,4}$
} 
${ }^{1}$ Department of Health Policy \& Management, ${ }^{2}$ Department of Epidemiology \& Biostatistics, and ${ }^{3}$ Department of Health Promotion \& Community Health Sciences, School of Rural Public Health, Texas A\&M Health Science Center, College Station, TX; and ${ }^{4}$ Department of Family \& Community Medicine, Scott \& White Healthcare, College of Medicine, Texas A\&M Health Science Center, Temple, TX

Patient Educ Couns 2014; 95: 111-17

\section{Background}

Individuals with any chronic disease, but especially those with diabetes, have multiple challenges they need to overcome to improve their health and their long-term outcomes. Diabetes education and support is one of the key methods to help patients self-manage their condition. One program created by researchers at Stanford University for in-person and online delivery is call Chronic Disease Self-management Program (CDSMP). It is a formalized and evidence-based peer-to-peer intervention that has been shown to improve diabetes outcomes. This study was designed to compare timeto-hospitalization among subjects enrolled in different diabetes self-management programs (DSMP) with the goal to determine whether the interventions delayed the occurrence of any acute event necessitating hospitalization.

\section{Methods}

Electronic medical records (EMR) were obtained for 376 adults enrolled in a randomized controlled trial (RCT) of type 2 diabetes (T2DM) self-management programs. All study participants had uncontrolled diabetes and were randomized into either personal digital assistant (PDA), Chronic Disease Self-Management Program (CDSMP), combined PDA and CDSMP (COM), or usual care (UC) groups. Subjects were followed for a maximum of 2 years. Time-to-hospitalization was measured as the interval between study enrollment and the occurrence of a diabetes-related hospitalization.

\section{Results}

Subjects enrolled in the CDSMP-only arm had significantly prolonged time-to-hospitalization (hazard ratio: $0.10 ; p=0.002$ ) when compared to subjects in the control arm. Subjects in the PDA-only and combined PDA and CDSMP arms showed no improvements in comparison to the control arm.

\section{Conclusions}

CDSMP can be effective in delaying time-to-hospitalization among patients with T2DM. Reducing unnecessary healthcare utilization, particularly inpatient hospitalization, is a key strategy to improving the quality of healthcare and lowering associated healthcare costs. The CDSMP offers the potential to reduce time-to-hospitalization among T2DM patients.

\section{Comment}

This study was an attempt to demonstrate if increasing the self-management knowledge, attitudes, skills, and behaviors of patients with type 2 diabetes would allow patients to be able to delay the time to be hospitalized for a diabetes-related condition. While those who received the self-management support without the addition of a PDA did better, it is promising that self-management support was effective. An important question not answered by this review is if the online version of the CDSMP might have had better results. That, however, must await the results from another study.

Note: Neal Kaufman has a potential conflict of interest with this study because of a strategic partnership with the developers of the CDSMP.

\section{Weight loss success in metabolic syndrome by telephone interventions: results from the SHINE study}

Weinstock $R S^{1}$, Trief $P M^{1}$, Cibula $D^{1}$, Morin $P C^{l}$, Delahanty $L M^{2}$

${ }^{1}$ SUNY Upstate Medical University, Syracuse, NY; and ${ }^{2}$ Massachusetts General Hospital and Harvard Medical School, Boston, MA

J Gen Intern Med 2013; 28: 1620-28

Note from author: The comments for this article can be seen after the next article.

\section{Background}

The evidence-based, NIH-funded Diabetes Prevention Program (DPP) was originally shown to lower the progression from prediabetes to diabetes by $58 \%$ when provided in person. To reach the millions of people at risk, there needs to be a variety of delivery approaches. Since its publication in 2002 , the DPP has been successfully delivered to adults with prediabetes via in-person groups and digitally. However, it hasn't been tested using group-based telephonic delivery.

\section{Methods}

Adults with a BMI $\geq 30 \mathrm{~kg} / \mathrm{m}^{2}$ and metabolic syndrome were recruited from primary care offices and randomized to receive the DPP delivered via conference calls (CC) or via individualized calls (IC). The Lifestyle Balance DPP curriculum was adapted for telephone delivery by educators chosen by the PCP practices included in the study. The 16week core curriculum was delivered weekly for the first 5 sessions, then monthly for the rest of year 1, and the additional postcore material was delivered during year 2. Registered dietitians provided individualized goal setting and feedback and worked with participants to overcome barriers to behavior change. Phone calls, both for IC and CC, were conducted at mutually agreed times. Participants were asked to self-weigh and to record diet and exercise in logbooks that were mailed to the coach and educator. The primary outcome was weight loss. Secondary outcomes such as waist circumference, fasting glucose, lipid, and blood pressure levels were also recorded.

\section{Results}

Five PCPs with diverse patient populations participated in this study. Most of the educators were nurses; 257 participants 
were randomized, 128 to the $\mathrm{CC}$ intervention and 129 to the IC intervention. Outcomes were recorded at baseline, 6 months, and 1 and 2 years. Participants in the $\mathrm{CC}$ intervention lost a mean of $4.5 \mathrm{~kg}$ at 6 months, $4.9 \mathrm{~kg}$ at 1 year, and $6.2 \mathrm{~kg}$ at 2 years. Participants in the IC intervention lost a mean of $4.3 \mathrm{~kg}$ at 6 months, $4.6 \mathrm{~kg}$ at 1 year, and $2.2 \mathrm{~kg}$ at 2 years (all $p<0.001)$. Mean weight loss at the end of the 2 year intervention was significantly greater in the $\mathrm{CC}$ arm $(p<0.001)$. In total, $43 \%$ of participants in the CC arm and $22 \%$ of participants in the IC arm reached the DPP goal of $7 \%$ weight loss by the end of the intervention. Waist circumference decreased in each group, but was not significantly different between the two groups. There was no significant changes in fasting glucose, lipid, or blood pressure levels.

\section{Conclusions}

PCP staff delivery of the DPP over the telephone results in significant weight loss in obese adults with metabolic syndrome. Sessions conducted by conference call were more effective in causing weight loss after 2 years of intervention compared to sessions conducted individually.

\section{A randomized comparative effectiveness trial of using cable television to deliver diabetes prevention programming}

Ackermann $R T^{l}$, Sandy $L G^{2}$, Beauregard $T^{2}$, Coblitz $M^{3}$, Norton $K L^{2}$, Vojta $D^{2}$

${ }^{1}$ Department of Medicine, Northwestern University Feinberg School of Medicine, Chicago, IL; ${ }^{2}$ UnitedHealth Center for Health Reform and Modernization, UnitedHealth Group, Minneapolis, MN; and ${ }^{3}$ Comcast Corporation, Philadelphia, PA

Obesity 2014; 22: 1601-7

\section{Background}

This article, along with the previous article on providing the DPP using the telephone, adds to the possible ways at-risk individuals can access the DPP program.

\section{Methods}

The authors performed an individually randomized, twoarm intervention trial with adults at risk for type 2 diabetes living in two U.S. cities. The study involved a 16-session lifestyle intervention delivered via "video-on-demand" cable television that was offered alone versus in combination with web-based lifestyle support tools. The major outcome measured was change in the participants' weight.

\section{Results}

A total of 306 individuals were randomized and offered the interventions. After 5 months, $265(87 \%)$ participants viewed at least 1 , and $110(36 \%)$ viewed $\geq 9$ of the video episodes. A total of $262(86 \%)$ participants completed a 5-month weight measurement. In intention-to-treat analysis with imputation of missing observations, mean weight loss at 5 months for both treatment groups combined was 3.3\% (95\% CI 0.7-5.0\%), regardless of intervention participation (with no differences between randomized groups $[p=0.19])$, and was $4.9 \%(95 \%$ CI $2.1-6.5 \%$ ) for participants who viewed $\geq 9$ episodes.

\section{Conclusions}

In-home delivery of evidence-based diabetes prevention programming in a reality television format, offered with or without online behavioral support tools, can achieve modest weight losses consistent with past implementation studies of face-to-face programs using similar content.

\section{Comment On The Previous Two Articles}

While some may think that an intervention using the oldfashioned telephone should not be included in a chapter on new technology, we disagree. The use of the telephone to facilitate participation in a group-based version of the NIH's Diabetes Prevention Program is an important addition to the other delivery approaches (i.e., communication channels) that have emerged over the past decade (e.g., cell phone and Internet).

This could also be true for "edutainment" programs (content that is designed to educate as well as to entertain) delivered via television. It is always good to see that television can actually improve someone's health when the vast majority of TV shows do the opposite. This creative approach to diabetes prevention has the potential to reach millions of people. It is hoped that the behavior change associated with watching the shows will be long lasting since the results were only at 5 months and most programs can get people to lose weight for 4-6 months. Prolonged weight loss is what really matters. If the researchers are able to follow the patients over time we may find out. I expect that the show will provide a modest level of improved behaviors at the individual level, but if they can reach very large numbers of people, there could be a significant population health effect.

Individuals should be able to access the education and support they need from whichever communication channel they prefer. Ideally, interventions based on the best research of what works for the specific targeted population (usually in-person) should be offered inperson, on the phone, via the cell phone, on the Internet, and via television delivered via cable and/or other means. All of the possible communication channels should be combined so that the benefits of each modality are optimized. Approaches should allow the seamless integration of the users' experiences and the real-time availability of data or information across the various channels. Participants should be able to choose which channel they prefer at that moment based on their unique characteristics, preferences, and circumstances.

\section{Self-directed interventions to promote weight loss: a systematic review of reviews}

Tang $J^{1}$, Abraham $C^{l}$, Greaves $C^{l}$, Yates $T^{2,3}$

${ }^{1}$ University of Exeter Medical School, Exeter, UK; ${ }^{2} N I H R$ Leicester-Loughborough Diet, Lifestyle, and Physical Activity Biomedical Research Unit, Leicester, UK; and ${ }^{3}$ Diabetes Research Centre, College of Medicine, Biological Sciences and Psychology, University of Leicester, Leicester, $U K$

J Med Internet Res 2014; 16: e58 


\section{Background}

Over the past years there have been a large number of weight management interventions that have used social media as part of or central to the program. The quality of and outcomes from these approaches have not been well documented. There is a critical need to evaluate these approaches to determine how they fit into the range of weight management approaches available to address the increasing prevalence of obesity worldwide.

\section{Methods}

The authors performed a systematic review of weightmanagement interventions that utilized online social media. PubMed, PsycINFO, and EMBASE were searched for articles published through March 25, 2013, that evaluated randomized control trials of online weight-related interventions using social media. Interventions were organized by those looking at weight management, diet, and physical activity. Descriptions of each intervention were provided, and participation levels were reported along with effectiveness of each intervention.

\section{Results}

A total of 517 studies were identified, of which 20 studies met the inclusion criteria. A meta-analysis was not done due to the variety of outcome measures and social media components used. Generally, use of social media was low. The most common types of social media used in online interventions were message boards and chat rooms. More mainstream forms of social media such as Facebook and Twitter were used in $15 \%$ of the studies and these were mostly studies published within the last few years. One study isolated the effect the use of social media component of the larger intervention had on outcomes and showed that use of social media improved weight-related outcomes but did not affect physical activity. This analysis showed that those with fewer social supports were more likely to use the social media component of the intervention and that use of social media was associated with less dropout.

\section{Conclusions}

This study gives an overview of the literature available, evaluating the use of social media in weight-related interventions. Since there are few studies that have looked specifically at the effect social media has on intervention results, the benefit of using social media in program delivery is unclear. Use of such media may provide social support and encourage adherence to behavioral change, but more studies are needed that evaluate the role social media plays in weight management.

\section{Comment}

This article looked at a growing and important approach to helping individuals lose weight-self-directed interventions in which the participant was able to experience the elements of the intervention without much (or any) need for professional interaction within the context of their everyday lives. These direct-to-consumer programs varied considerably and, not surprisingly, had a variety of outcomes, some of which we find quite promising.

In addition to self-directed approaches, one key related element that is often overlooked in interventions provided by clinicians is the important concept of selftailoring. The ability of individuals to pick and choose what education and support they need and when they need it has been shown to improve outcomes.

It will be important to determine how these selfdirected approaches and interventions involving personal contact or online support can be mutually reinforcing. These findings are not just relevant for weight loss, an area with a long-standing and very robust consumer focus. Direct-to-consumer approaches for nearly all conditions are in many ways in competition with clinically linked programs, and ideally they should be integrated or at least provide a reinforcing function.

\section{Are health behavior change interventions that use online social networks effective? A systematic review}

Maher $C A^{1}$, Lewis $L K^{1}$, Ferrar $K^{2}$, Marshall $S^{3}$, De Bourdeaudhuij $I^{4}$, Vandelanotte $C^{5}$

${ }^{1}$ Health and Use of Time Group and ${ }^{2}$ Exercise for Health and Human Performance Group, University of South Australia, Adelaide, Australia; ${ }^{3}$ School of Medicine, University of California, San Diego, CA; ${ }^{4}$ Department of Movement and Sports Sciences, University of Ghent, Ghent, Belgium; and ${ }^{5}$ Institute for Health and Social Sciences, Central Queensland University, North Rockhampton, Australia

J Med Internet Res 2014; 16: e40

Note from author: The comments for this article can be seen after the next article.

\section{Background}

The dramatic growth of Web 2.0 technologies and online social networks offers immense potential for the delivery of health behavior change campaigns. However, it is currently unclear how online social networks may best be harnessed to achieve health behavior change. The intent of the study was to systematically review the current level of evidence regarding the effectiveness of online social network health behavior interventions.

\section{Methods}

A comprehensive search of the literature was done to identify relevant articles. Reports of intervention effectiveness were summarized, and effect sizes were calculated wherever possible. Attrition, engagement, and fidelity (actual usage/intended usage) with the social networking component of the interventions were scrutinized.

\section{Results}

Ten studies met inclusion criteria and involved a total of 113,988 participants (ranging from $n=10$ to $n=107,907$ ). Interventions included commercial online health social 
network websites $(n=2)$, research health social network websites $(n=3)$, and multicomponent interventions delivered in part via preexisting popular online social network websites (Facebook $n=4$ and Twitter $n=1$ ). Nine of the 10 included studies reported significant improvements in some aspect of health behavior change or outcomes related to behavior change. Effect sizes for behavior change ranged widely from -0.05 to 0.84 , but in general were small in magnitude and statistically nonsignificant. Participant attrition ranged from $0 \%$ to $84 \%$. Engagement and fidelity were relatively low, with most studies achieving 5-15\% fidelity (with one exception, which achieved 105\% fidelity).

\section{Conclusions}

To date, there is very modest evidence that interventions incorporating online social networks may be effective; however, this field of research is in its infancy. Further research is needed to determine how to maximize retention and engagement, whether behavior change can be sustained in the longer term, and how to exploit online social networks to achieve mass dissemination.

\section{The role of social media in online weight management: systematic review}

Chang T, Chopra V, Zhang C, Woolford SJ

University of Michigan, Ann Arbor, MI

J Med Internet Res 2013; 15: e262

\section{Background}

Social media applications are promising adjuncts to online weight management interventions through facilitating education, engagement, and peer support. However, the precise impact of social media on weight management is unclear. The objective of this study was to systematically describe the use and impact of social media in online weight management interventions.

\section{Methods}

After a thorough search of the literature, randomized controlled trials of online weight management interventions that included a social media component for individuals of all ages were selected.

\section{Results}

Twenty studies met eligibility criteria. All study participants were adults. Although message boards and chat rooms were the most common social media component included, their effect on weight outcomes was not reported in most studies. Only one study measured the isolated effect of social media. It found greater engagement of participants, but no difference in weight-related outcomes. In all, $65 \%$ of studies were of high quality; $15 \%$ of studies were at low risk of bias.

\section{Conclusions}

Despite the widespread use of social media, few studies have quantified the effect of social media in online weight management interventions; thus, its impact is still unknown. Although social media may play a role in retaining and en- gaging participants, studies that are designed to measure its effect are needed to understand whether and how social media may meaningfully improve weight management.

\section{Comments For The Above Two Articles}

These two articles, among others in this chapter, try to summarize the state of the art regarding the impact of social networking on the outcomes that matter. The bottom line is that it is way too soon to tell. As is true with all information technology, but especially with social networking, the extraordinarily rapid pace of improvement makes it exceedingly difficult to understand what is actually being provided, to whom, how, and with what dose. This reality, coupled with the variation in social networking use by age, gender, ethnicity, socioeconomic status, etc., makes any real and meaningful assessment nearly impossible. This, however, should not deter anyone interested in improving health outcomes. There are some basic principles that hold regardless of the technology. We human beings are basically social animals, and even those among us who are less social need and benefit from interactions with others. This is true no matter the delivery mechanism...no matter the communication channel. The key will be to allow each person to self-tailor his or her preferred approach that can certainly change in an instant or over longer periods of time. The key lesson for healthcare providers is that patients should be able to receive the education and support they need via whichever communication channel they prefer and within a time frame that makes sense to them. These experiences should be integrated such that what happens via one communication channel informs what transpires in another. At a minimum, the data entered in one approach should be available in all channels.

\section{DietBet: a web-based program that uses social gaming and financial incentives to promote weight loss}

Leahey $T^{l}$, Rosen $J^{2}$

${ }^{I}$ Department of Psychiatry and Human Behavior, Brown Medical School, The Miriam Hospital's Weight Control and Diabetes Research Center, Providence, RI; and ${ }^{2}$ DietBet, Inc., New York, NY

JMIR Serious Games 2014; 2: e2

\section{Background}

There is reason to believe that providing financial incentives has the capacity to increase patient motivation to participate in programs designed to prevent or treat chronic disease. This could also be said for using social gaming. However, there is not sufficient evidence that either, or both in combination, can make a real difference in engagement and outcomes over time.

\section{Methods}

Program description: The DietBet gaming platform allows people to enter money in a betting pool upon enrollment, and to interact with other players. Players are encouraged to post 
to the online message board, along with their weigh-in results. All players who lose $4 \%$ of their weight by the end of 4 weeks split the money pool. Games are either open or closed access, where players all know each other.

The study hypothesized that betting more money at enrollment, completing more weigh-ins, and having greater social engagement/influence would result in greater weight loss. The study also looked at whether weight loss outcomes were associated with game characteristics such as number of players.

\section{Results}

From December 2012 to July 2013, 1934 DietBet games were completed that included 39,387 participants. Players were predominantly female with an average starting weight of $87.8 \mathrm{~kg}$. $65.63 \%$ of participants completed the official endof-game weigh-in. Using intention-to-treat analysis, average weight loss at 4 weeks was $2.6 \%$. In total, $43.6 \%$ of enrollees lost $4 \%$ of their body weight by the end of their 4 -week game, with 5268 participants achieving $5 \%$ weight loss. Those who lost more weight tended to be male, weighed less at baseline, bet more money, weighed in more often, shared game participation on Facebook, and were more active in posting on the DietBet platform. Weight loss seemed to cluster among participants within the same game, so weight loss seems to be influenced by that of other participants within the same game, and larger games were associated with greater weight loss. Closed games did not seem to be different from open games in terms of weight loss outcomes.

\section{Conclusions}

This study evaluated the effects of DietBet, and the results support the idea that an online social gaming program can incentivize clinically significant amounts of short-term weight loss.

\section{Comment}

This study was included mainly because of its novel use of social gaming and financial incentives in one package. While lacking scientific rigor (e.g., no control or comparison group), we can still learn from the results. People are motivated by financial incentives, but most of the evidence would seem to support an impact on early engagement without long-term change. This approach, with social aspects tied to financial incentives, might be one that would be able to generate sustainable behavior change. That would require a much longer and methodologically sound study.

Using the Internet to help with diet, weight, and physical activity: results from the Health Information National Trends Survey (HINTS)

McCully SN, Don BP, Updegraff JA

Department of Psychology, Kent State University, Kent, $\mathrm{OH}$

J Med Internet Res 2013; 15: e148

\section{Background}

Internet use for diet, weight, and physical activity (DWPA) is becoming more prevalent. Understanding this trend and the relationship between use and adherence to DWPA behaviors can improve approaches to the delivery of Internet-based content and interventions.

\section{Methods}

This study looks at changes in use of the Internet for DWPA programs between 2007 and 2011 using data from the U.S. National Cancer Institute's Health Information National Trends Survey (HINTS). HINTS is a national probability survey looking at health information access and understanding. 3582 participants completed the 2007 survey and 3959 completed the 2011 survey.

\section{Results}

Study results showed that Internet use for DWPA has increased from 2007 to 2011 (OR 1.05, 95\% CI 0.99-1.12, $p=0.07)$. Those who used the Internet for DWPA were more likely to be younger, more educated, married, with higher BMIs, and nonwhite. Men are currently as likely as women to use Internet-based DWPA programs, although since 2007 demographic trends have shown increase in younger, single, female, and less educated Internet DWPA users. Use has increased among Hispanics and has decreased among non-Hispanic blacks. Users reported consuming an average of 2.54 cups of fruit and 2.88 cups of vegetables each day, and exercising 2.81 days per week.

\section{Conclusions}

This study using the HINTS survey results shows demographic trends in Internet use for DWPA in 2011, and evaluates the changes in use between 2007 and 2011 in terms of gender, BMI, age, education, race, and marital status. Users in 2011 met recommendations for fruit and vegetable intake but not for physical activity.

\section{Comment}

This article reports on data from a nationally representative survey to inform Internet-based DWPA program developers and evaluators about the trends in usage. The fact that use of the Internet for DWPA has increased supports the idea that the Internet is increasingly useful as a tool to disseminate DWPA programs and interventions. Such programs currently seem to support healthy fruit and vegetable consumption, but programs that effectively address physical activity are still sorely needed. The study results show that user demographics have great fluctuation, and this emphasizes the fact that Internet programs have to be tailored to meet the needs of their target populations.

\section{Effects of a web-based intervention on physical activity and metabolism in older adults: randomized controlled trial}

Wijsman $C^{1}$, Westendorp RGJ $J^{1,2}$, Verhagen $E A L M^{3}$,
Catt $M^{4}$, Slagboom PE $E^{5}$, de Craen AJM $M^{1,6}$,
Broekhuizen $K^{7}$, van Mechelen $W^{3}$, van Heemst $D^{1,6}$,
van der Ouderaa $F^{2,6}$, Mooijaart $S P^{1,6}$


${ }^{I}$ Department of Gerontology and Geriatrics, Leiden University Medical Center, Leiden, The Netherlands; ${ }^{2}$ Leyden Academy of Vitality and Ageing, Leiden, The Netherlands; ${ }^{3}$ Department of Public and Occupational Health, EMGO+ Institute, VU University Medical Center, Amsterdam, The Netherlands; ${ }^{4}$ Institute for Ageing and Health, Newcastle University, Newcastle upon Tyne, UK; ${ }^{5}$ Department of Medical Statistics, Molecular Epidemiology Section, Leiden University Medical Center, Leiden, The Netherlands; ${ }^{6}$ Netherlands Consortium for Healthy Ageing, Leiden, The Netherlands; and ${ }^{7}$ Institute for Evidence-Based Medicine in Old Age, IEMO, Leiden, The Netherlands

J Med Internet Res 2013; 15: e233

\section{Background}

Lack of physical activity can lead to significant negative impacts on a person's health and well-being. Examples include changes in metabolism, decreases in functional abilities, and increased risk for a range of chronic diseases in old age. The potential of Web-assisted programs to increase physical activity and improve metabolism in older individuals holds promise.

\section{Methods}

The authors conducted a 3-month randomized, waitlistcontrolled trial in a volunteer sample of 235 inactive adults aged 60-70 years without diabetes. The intervention group received the Internet program Philips DirectLife, which targeted increasing physical activity using accelerometer-based monitoring and feedback and digital coaching. The primary outcome was increase in physical activity measured using ankle- and wrist-worn accelerometers. Secondary outcomes included changes in weight, waist circumference and body fat, and parameters of glucose metabolism.

\section{Results}

226 participants (97\%) completed the 3 month study. Activity counts increased by $46 \%$ in the intervention group, compared to $12 \%$ in the control group $(P<.001)$ when measured at the ankle. Measured at the wrist, activity counts increased by $11 \%$ in the intervention group and $5 \%$ in the control group $\left(P_{\mathrm{d}}=.11\right)$. This increase corresponded to an estimated daily increase of 11 minutes in moderate-to-vigorous activity in the intervention group versus 0 minutes in the control group $(P=.001)$. Weight decreased significantly more in the intervention group compared to controls $(-1.5 \mathrm{~kg}$ vs $-0.8 \mathrm{~kg}$ respectively, $P=.046)$, as did waist circumference $(-2.3 \mathrm{~cm}$ vs $-1.3 \mathrm{~cm}$ respectively, $P=.036)$ and fat mass ( $-0.6 \%$ vs $0.07 \%$ respectively, $P=.025)$. Insulin and $\mathrm{HbA} 1 \mathrm{c}$ levels were significantly more reduced in the intervention group compared to controls (both $P<.05$ ).

\section{Conclusions}

This study demonstrated that in inactive older adults, a 3-month Web-based physical activity intervention consisting of accelerometry and digital coaching was effective in increasing daily physical activity and improving metabolic health. Web-based interventions could provide opportunities for large scale prevention of obesity and obesity-related co- morbidities such as Metabolic Syndrome and type 2 diabetes in older people.

\section{Comment}

These are promising, but quite early, results from a very short intervention. But let's celebrate any good results we can. Nearly any program that pays attention and provides support to sedentary and overweight adults can get results for a few months. It is hoped that the gains can be maintained. However, I take great exception with the authors. Since when are people aged 60-70 considered "older adults"? Don't they know the 60s are the new 50s? With life expectancy increasing, at least in the highresource part of the world, an older individual is anyone older than the majority of people in the room. And when I look at the rooms I am in, that is much older than 65 or 70. Perhaps I hang out with the wrong crowd.

\section{The impact of mobile health interventions on chronic disease outcomes in developing countries: a systematic review}

Beratarrechea $A^{1}$, Lee $A G^{2}$, Willner $J M^{2}$, Jahangir $E^{2}$, 'n Ciapponi $A^{l}$, Rubinstein $A^{1}$

${ }^{1}$ South American Center of Excellence for Cardiovascular Health, Institute for Clinical Effectiveness and Health Policy, Buenos Aires, Argentina; and ${ }^{2}$ Fogarty International Center, Bethesda, MD

Telemed J e-Health 2014; 20: 75-82

\section{Background}

Rates of chronic diseases will continue to rise in developing countries unless effective and cost-effective interventions are implemented. Technologies created for high-resource countries are often not affordable or scalable in low-resource countries. In addition, solutions generated and tested with the challenge of lack of Internet access and the requirement to be practical for a variety of settings can often provide much needed innovation for programs in higher resource countries. This review's aim was to discuss the impact of mobile health ( $\mathrm{m}$-health) on chronic disease outcomes in low- and middleincome countries (LMIC).

\section{Methods}

Systematic literature searches were performed to identify controlled studies evaluating cell phone voice and text message interventions to address chronic diseases in adults in LMIC. Outcomes measured included morbidity, mortality, hospitalization rates, behavioral or lifestyle changes, process of care improvements, clinical outcomes, costs, patientprovider satisfaction, compliance, and health-related quality of life (HRQoL).

\section{Results}

Nine randomized controlled trials with 4,604 participants were reviewed. Most of the studies addressed more than one outcome. Six studied clinical outcomes, six studied processes of care, three examined healthcare costs, and two examined 
HRQoL. M-health positively impacted chronic disease outcomes, improving attendance rates, clinical outcomes, and HRQoL, and was cost-effective.

\section{Conclusions}

M-health is emerging as a promising tool to address access, coverage, and equity gaps in developing countries and lowresource settings. The results for $m$-health interventions showed a positive impact on chronic diseases in LMIC. However, a limiting factor of this review was the relatively small number of studies and patients enrolled, highlighting the need for more rigorous research in this area in developing countries.

\section{Comment}

The challenges to improving outcomes for people with diabetes and other chronic disease in low-resource countries are immense. While mHealth demonstrates tremendous promise, that promise will only be realized when there is enough experience with the variety of approaches. This review of programs with some research rigor can help lead the way, but much more needs to be done. It is highly unlikely-nearly impossible-for an approach that works in one country, with one population within that country, to be expected to work in other populations. While the principles at the core that help people manage their conditions are similar across populations, the exact approaches must be tailored for each population. One key element that is often overlooked is the concept of self-tailoring, the ability of the individual to pick and choose what education and support they need and when they need it. That element should be included whenever possible in all interventions.

\section{Effectiveness of mobile phone messaging in prevention of type 2 diabetes by lifestyle modification in men in India: a prospective, parallel-group, randomized controlled trial}

Ramachandran $A^{1}$, Snehalatha $C^{l}$, Ram $J^{1}$, Selvam $S^{1}$, Simon $M^{l}$, Nanditha $A^{l}$, Shetty $A S^{l}$, Godsland $I F^{2}$, Chaturvedi $N^{2}$, Majeed $A^{2}$, Oliver $N^{2}$, Toumazou $C^{3}$, Alberti $K G^{3}$, Johnston $D G^{3}$

${ }^{1}$ India Diabetes Research Foundation and Dr. A. Ramachandran's Diabetes Hospitals, Chennai, India; and ${ }^{2}$ Faculty of Medicine and ${ }^{3}$ Faculty of Engineering, Imperial College, London, UK

Lancet Diabetes Endocrinol 2013; 1: 191-98

\section{Background}

Mobile text messaging has the potential to inexpensively provide education and support to individuals with a variety of chronic conditions. They could be effective for individuals with a range of financial and other barriers to intensive interventions. They could also be used to provide in-themoment assessment and intervention. Studies are needed to determine the effectiveness of these approaches with specific populations - in this case, working men with impaired glucose tolerance in India.

\section{Methods}

Working men with impaired glucose tolerance in southeast India were randomized to a mobile phone messaging intervention or standard care (control). Intervention duration was 2 years. Messages were delivered at frequent intervals and consisted of information about healthy lifestyle, cues to start physical activity and healthy eating, as well as strategies and motivational messages to encourage maintenance of healthy diet and exercise. Content at a given time was based off of the trans-theoretical model of behavioral change. Oral glucose tolerance tests were performed at baseline, 12 months, and 24 months. Intervention acceptability was evaluated by questionnaire.

\section{Results}

A total of 537 participants were included in the study. Text messaging was requested to be delivered at a median of 18 times/month at the start of the intervention and dropped to 12 times/month by the end of 2 years. A total of $18 \%$ of participants receiving mobile phone messaging intervention developed type 2 diabetes within the 2 year intervention period compared to $27 \%$ in the control group. The number needed to treat to prevent one case of diabetes was 11 (95\% CI: 6-55). The intervention was overall described as welcome.

\section{Conclusions}

Mobile phone messaging can be an effective tool to deliver lifestyle interventions to prevent type 2 diabetes in high-risk individuals.

\section{Comment}

This is a very well-done and important study demonstrating that with appropriate mobile phone messages, individuals at high risk for developing type 2 diabetes can change their behaviors and lower their risk. Many studies have demonstrated that if people who are at risk eat better and are more active, they can lower their risk. The key is demonstrating a cost-effective intervention that works with a specific target population to help the participants change their behaviors enough to get the desired results. This study does just that. Once an intervention is proven effective, the really hard work starts. There must be an understanding of how to bring a program to scale with an appropriate delivery system of some kind. And of course, there must be a way to pay for it. It will be interesting to see if there will be a way to bring the intervention to the millions of people who need it.

\section{Conclusions}

This year's articles, while worth reviewing and reading, nonetheless don't provide a good-enough sense of how information technology can and will be transformative in the way we provide education and support to individuals with, or at risk for, diabetes. This is not surprising given the extraordinary diversity of human existence and the variety of behaviors that fall within the scope of diabetes prevention and treatment. 
In an effort to understand how to move the field forward, and to help decide what articles would be ideal to be included in this article in the future, I found an article by Baker et al. quite helpful (1). They describe two types of strategies needed to move the field of eHealth forward-those that improve the efficiency of eHealth interventions, and those that improve its quality.

Efficiency strategies include

1. think small and conduct studies that can target discrete but significant questions;

2. use efficient designs such as quasi-experimental designs and surrogate endpoints, and experimentally modify and evaluate interventions and delivery systems already in use;

3. study universal issues by focusing on timeless behavioral, psychological, and cognitive principles and systems;

4. anticipate the next big thing by listening to voices outside normal practice and connect different perspectives for new insights;

5. improve information and data delivery systems; and

6. develop models that explain how interventions work and identify factors that modulate how well they work.

Quality strategies include

1. strive for continuous quality improvement incorporating clinical and technological progress;

2. help consumers and clinicians easily identify quality;

3 . reduce the costs of care; and
4. deeply understand users in order to deliver what they want and need.

These approaches get at the fundamental drivers of outcomes regardless of the specifics of the studied intervention. With technology evolving at such a rapid pace, true advances will be primarily determined by the answers to these types of questions. They will not come from large randomized controlled trails that are often technologically obsolete before the results of the studies are published. It is hoped that more studies are designed to address these foundational issues.

\section{Author Disclosure Statement}

N.K. has no conflicts with any of the reviewed articles with the exception of the article by Adepoju et al. His potential conflict with this study is because his company (DPS Health) has a strategic partnership with the developers of the CDSMP. He is founder, co-owner, and chief medical officer of DPS Health, a software development and population health management company based in Los Angeles, CA. DPS Health creates and distributes web- and cell phone-based weight management and self-management support services.

R.R.B. has no conflict of interest to disclose.

\section{Reference}

1. Baker TB, Gustafson DH, Shah D. How can research keep up with eHealth? Ten strategies for increasing the timeliness and usefulness of eHealth research. $\underline{J \text { Med Internet Res }}$ 2014; 16: e36. 


\section{This article has been cited by:}

1. Crangle Colleen E. , MSc, PhD , 1 Bradley Colin , MD, MB , 2 Carlin Paul F. , BSc, MPA , 3 Esterhay Robert J. , MD, BA , 1 Harper Roy , MD, BSc , 4 Kearney Patricia M. , MB, MPH, PhD , 5 Lorig Kate , MS, DrPH , 6 McCarthy Vera J.C. , MA, PhD , 7 McTear Michael F. , MA, PhD , 8 Tuttle Mark S. , AB, BE, FACMI , 9 Wallace Jonathan G. , BSc, MSc , 8 and Savage Eileen, PhD, MEd 7 1School of Public Health and Information Sciences, University of Louisville, Louisville, Kentucky. 2Department of General Practice, University College Cork, Cork, Ireland. 3South Eastern Health and Social Care Trust, Research Office, Dundonald, Northern Ireland, United Kingdom. 4Department of Endocrinology and Diabetes, The Ulster Hospital, South Eastern Health and Social Care Trust, Dundonald, Northern Ireland, United Kingdom. 5Department of Epidemiology and Public Health, University College Cork, Cork, Ireland. 6Patient Education Research Center, Stanford University School of Medicine, Stanford, California. 7School of Nursing and Midwifery, University College Cork, Cork, Ireland. 8School of Computing and Mathematics, University of Ulster, Jordanstown, Northern Ireland, United Kingdom. 9Apelon, Hartford, Connecticut. . 2017. Soliciting and Responding to Patients' Questions about Diabetes Through Online Sources. Diabetes Technology \& Therapeutics 19:3, 194-199. [Abstract] [Full Text HTML] [Full Text PDF] [Full Text PDF with Links] 